\title{
HYBRID CHAOS SYNCHRONIZATION OF FOUR-SCROLL SYSTEMS VIA ACTIVE CONTROL
}

\author{
Rajagopal Karthikeyan * - Vaidyanathan Sundarapandian ${ }^{* *}$
}

\begin{abstract}
This paper investigates the hybrid chaos synchronization of identical Wang four-scroll systems (Wang, 2009), identical Liu-Chen four-scroll systems (Liu and Chen, 2004) and non-identical Wang and Liu-Chen four-scroll systems. Active control method is the method adopted to achieve the hybrid chaos synchronization of the four-scroll chaotic systems addressed in this paper and our synchronization results are established using Lyapunov stability theory. Since the Lyapunov exponents are not required for these calculations, the active control method is effective and convenient to hybrid synchronize identical and different Wang and Liu-Chen four-scroll chaotic systems. Numerical simulations are also shown to illustrate and validate the hybrid synchronization results derived in this paper.
\end{abstract}

K e y w o r d s: active control, chaos, chaotic systems, four-scroll systems, Wang system, Liu-Chen system

\section{INTRODUCTION}

Chaotic systems are nonlinear dynamical systems that are highly sensitive to initial conditions. This sensitivity is popularly known as the butterfly effect [1]. The chaos phenomenon was first observed in weather models by Lorenz $([2], 1963)$. Chaos is an interesting nonlinear phenomenon and it has been intensively and extensively studied in the last three decades. Chaos theory has wide applications in several fields such as physical systems [3], chemical systems [4], ecological systems [5], secure communications [6-8], etc.

Synchronization of chaotic systems is a phenomenon that may occur when two or more chaotic oscillators are coupled or when a chaotic oscillator drives another chaotic oscillator. Because of the butterfly effect which causes the exponential divergence of the trajectories of two identical chaotic systems started with nearly the same initial conditions, synchronizing two chaotic systems is seemingly a very challenging research problem.

In most of the chaos synchronization approaches, the master-slave or drive-response formalism is used. If a particular chaotic system is called the master or drive system and another chaotic system is called the slave or response system, then the idea of synchronization is to use the output of the master system to control the slave system so that the states of the slave system track the states of the master system asymptotically.

Since the seminal work by Pecora and Carroll ([9], 1990) on complete synchronization of chaotic systems, several approaches have been developed for chaos synchronization such as the OGY method [10], sampled-data feedback synchronization method [11], the time-delay feedback method $[12,13]$, the active control method [1418], the adaptive control method [19-23], the backstep- ping method [24-26], the sliding mode control method [27-30] and others.

So far, various synchronization methods have been developed such as the complete synchronization [9], the phase synchronization [31], the generalized synchronization [32], the anti-synchronization [33-36], the projective synchronization [37] and the generalized projective synchronization [38-40].

Complete synchronization (CS) is characterized by the equality of state variables evolving in time, while the antisynchronization (AS) is characterized by the disappearance of the sum of relevant state variables evolving in time. Projective synchronization (PS) is characterized by the fact the master and slave systems could be synchronized up to a scaling factor, whereas in generalized projective synchronization (GPS), the responses of the synchronized dynamical states synchronize up to a constant scaling matrix $\alpha$. It is easy to see that the complete synchronization and the anti-synchronization are the special cases of the generalized projective synchronization where the scaling matrix $\alpha=I$ and $\alpha=-I$, respectively.

In hybrid synchronization of chaotic systems [41], one part of the systems (for instance, the odd-numbered states) is completely synchronized, while the other part (for instance, the even numbered states) is anti-synchronized so that complete synchronization (CS) and antisynchronization (AS) co-exist in the master-slave chaotic systems. The co-existence of CS and AS is very useful in secure communication and chaotic encryption schemes.

In this paper, we apply the active control method to derive new results for the hybrid synchronization of identical Wang four-scroll chaotic systems ([42], 2009), identical Liu-Chen four-scroll chaotic systems ([43], 2004) and non-identical Wang and Liu-Chen four-scroll chaotic systems. Our hybrid synchronization results for the four-

\footnotetext{
* Department of ECE, Velammal Institute of Technology, Panchetti, Tiruvalluvar District, India, rkarthiekeyan@gmail.com ** Research and Development Centre, Vel Tech Dr. RR \& Dr. SR Technical University, Avadi, Chennai-600 062, India, sundarvtu@gmail.com
} 


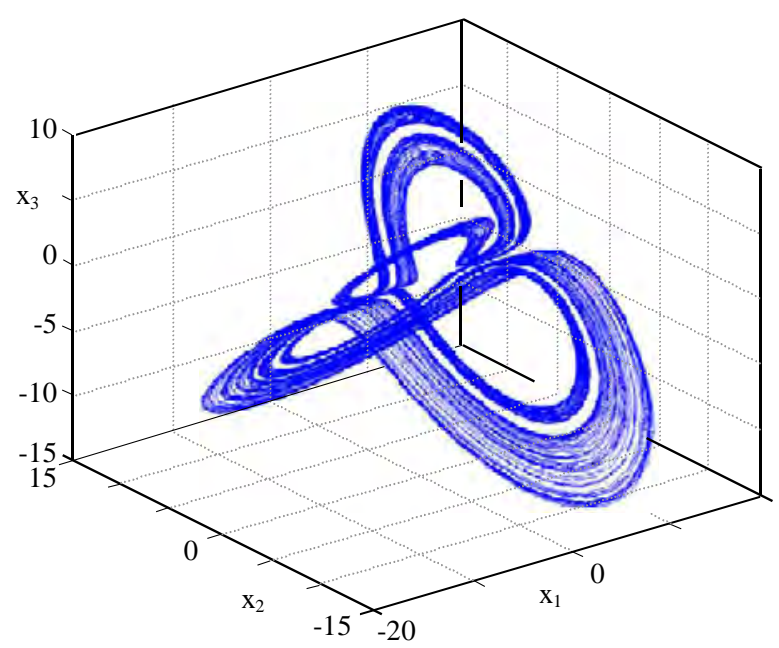

Fig. 1. The four-scroll attractor of the Wang system

scroll chaotic systems are established by the Lyapunov stability theory [44].

This paper has been organized as follows. In Section 2, we give a description of the four-scroll chaotic systems addressed in this paper. In Section 3, we discuss the hybrid synchronization of identical Wang four-scroll systems (2009). In Section 4, we discuss the hybrid synchronization of identical Liu-Chen four-scroll systems (2004). In Section 5, we discuss the hybrid synchronization of nonidentical Wang and Liu-Chen four-scroll systems. In Section 6 , we summarize the main results obtained in this paper.

\section{SYSTEMS DESCRIPTION}

The Wang four-scroll system is a new chaotic system derived by L. Wang ([42], 2009).

The Wang system is described by the 3D dynamics

$$
\begin{aligned}
& \dot{x}_{1}=a\left(x_{1}-x_{2}\right)-x_{2} x_{3}, \\
& \dot{x}_{2}=-b x_{2}+x_{1} x_{3}, \\
& \dot{x}_{3}=-c x_{3}+d x_{1}+x_{1} x_{2}
\end{aligned}
$$

where $x_{1}, x_{2}, x_{3}$ are the states and $a, b, c, d$ are positive, constant parameters of the system.

The system (1) is chaotic when the parameter values are taken as

$$
a=1, \quad b=5.7, c=5 \text { and } d=0.06 .
$$

Figure 1 depicts the four-scroll attractor of the Wang chaotic system (1).

The Liu-Chen four-scroll system is a new chaotic system derived by W. Liu and G. Chen ([43], 2004). The Liu-Chen system is described by the $3 \mathrm{D}$ dynamics

$$
\begin{aligned}
& \dot{x}_{1}=\alpha x_{1}-x_{2} x_{3}, \\
& \dot{x}_{2}=-\beta x_{2}+x_{1} x_{3}, \\
& \dot{x}_{3}=-\gamma x_{3}+x_{1} x_{2}
\end{aligned}
$$

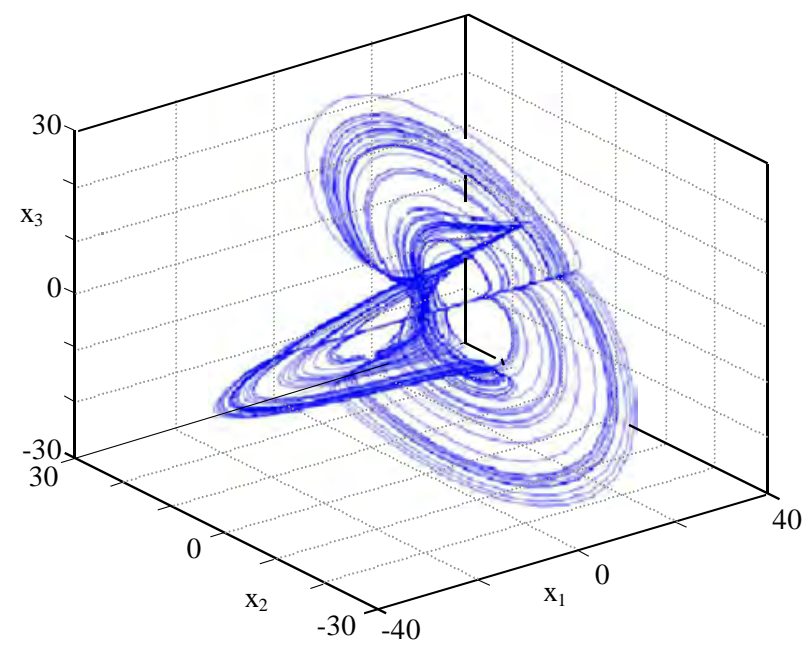

Fig. 2. The four-scroll attractor of the Liu-Chen system

where $x_{1}, x_{2}, x_{3}$ are the states and $\alpha, \beta, \gamma$ are positive, constant parameters of the system. The system (2) is chaotic when the parameter values are taken as

$$
\alpha=0.4, \quad \beta=12 \text { and } \gamma=5 \text {. }
$$

Figure 2 depicts the four-scroll attractor of the LiuChen chaotic system (2).

\section{HYBRID SYNCHRONIZATION OF THE IDENTICAL WANG FOUR-SCROLL SYSTEMS}

\subsection{Theoretical Results}

In this section, we deploy the active control method to derive new results for the hybrid synchronization of identical Wang four-scroll systems ([42], 2009).

Thus, the master system is described by the Wang dynamics

$$
\begin{aligned}
& \dot{x}_{1}=a\left(x_{1}-x_{2}\right)-x_{2} x_{3}, \\
& \dot{x}_{2}=-b x_{2}+x_{1} x_{3}, \\
& \dot{x}_{3}=-c x_{3}+d x_{1}+x_{1} x_{2}
\end{aligned}
$$

where $x_{1}, x_{2}, x_{3}$ are the states and $a, b, c, d$ are positive, constant parameters of the system.

The slave system is described by the controlled Wang dynamics

$$
\begin{aligned}
& \dot{y}_{1}=a\left(y_{1}-y_{2}\right)-y_{2} y_{3}+u_{1}, \\
& \dot{y}_{2}=-b y_{2}+y_{1} y_{3}+u_{2} \\
& \dot{y}_{3}=-c y_{3}+d y_{1}+y_{1} y_{2}+u_{3}
\end{aligned}
$$

where $y_{1}, y_{2}, y_{3}$ are the states and $u_{1}, u_{2}, u_{3}$ are the active controllers to be designed. 

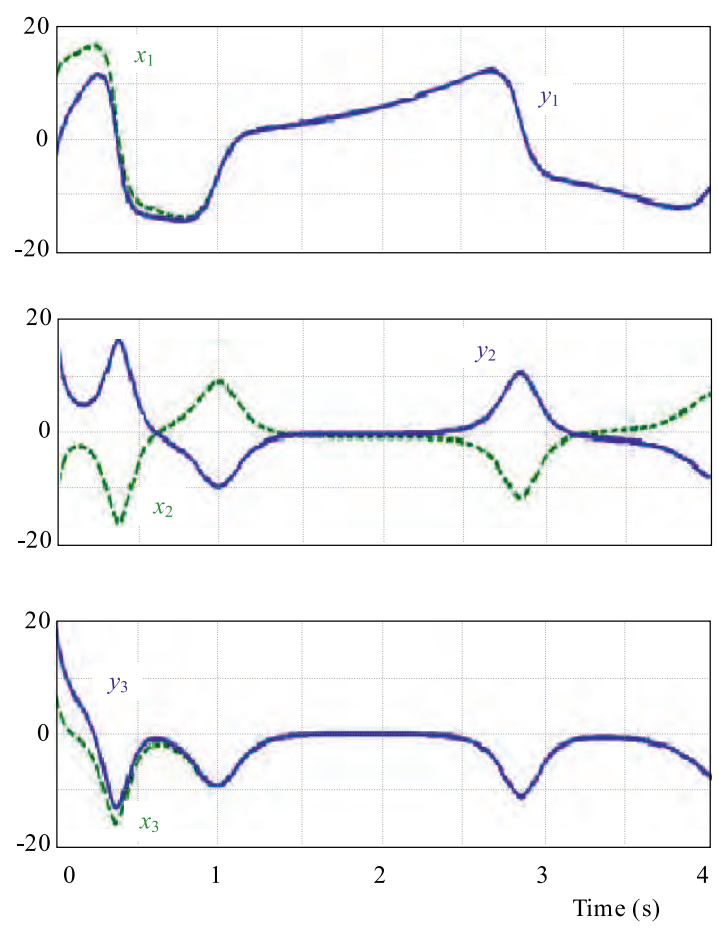

Fig. 3. Hybrid synchronization of the identical Wang four-scroll systems

The hybrid synchronization error is defined as

$$
\begin{aligned}
& e_{1}=y_{1}-x_{1}, \\
& e_{2}=y_{2}+x_{2}, \\
& e_{3}=y_{3}-x_{3} .
\end{aligned}
$$

A simple calculation gives the error dynamics as

$$
\begin{aligned}
& \dot{e}_{1}=a\left(e_{1}-e_{2}+2 x_{2}\right)+u_{1}, \\
& \dot{e}_{2}=-b e_{2}+y_{1} y_{3}+x_{1} x_{3}+u_{2}, \\
& \dot{e}_{3}=-c e_{3}+d e_{1}+y_{1} y_{2}-x_{1} x_{2}+u_{3} .
\end{aligned}
$$

We define the active controls $u_{1}(t), u_{2}(t)$ and $u_{3}(t)$ as

$$
\begin{aligned}
& u_{1}(t)=-a\left(e_{1}-e_{2}+2 x_{2}\right)+y_{2} y_{3}-x_{2} x_{3}-k_{1} e_{1} \\
& u_{2}(t)=b e_{2}-y_{1} y_{3}-x_{1} x_{3}-k_{2} e_{2}, \\
& u_{3}(t)=c e_{3}-d e_{1}-y_{1} y_{2}+x_{1} x_{2}-k_{3} e_{3}
\end{aligned}
$$

where $k_{1} k_{2}, k_{3}$ are positive constants.

Substituting (7) into (6), the error dynamics simplifies to

$$
\begin{aligned}
& \dot{e}_{1}=-k_{1} e_{1}, \\
& \dot{e}_{2}=-k_{2} e_{2}, \\
& \dot{e}_{3}=-k_{3} e_{3} .
\end{aligned}
$$

TheOREM 1. The identical Wang four-scroll systems (3) and (4) with constant, positive parameters are globally and exponentially hybrid-synchronized by the active control law (7), where the gains $k_{1}, k_{2}, k_{3}$ are positive constants.

Pr o of. This result is a simple consequence of the Lyapunov stability theory. by

Consider the quadratic Lyapunov function $V$ defined

$$
V\left(e_{1}, e_{2}, e_{3}\right)=\frac{1}{2}\left(e_{1}^{2}+e_{2}^{2}+e_{3}^{2}\right),
$$

which is a positive definite function on $R^{3}$.

Differentiating $V$ along the trajectories of (8), we get

$$
\dot{V}=-k_{1} e_{1}^{2}-k_{2} e_{2}^{2}-k_{3} e_{3}^{2},
$$

Clearly, $\dot{V}$ is a negative definite function on $R^{3}$. Hence, by the Lyapunov stability theory [44], it follows that $e_{i}(t) \rightarrow 0$ as $t \rightarrow \infty$ for $i=1,2,3$. This completes the proof.

\subsection{Numerical Results}

For the numerical simulations, the fourth-order RungeKutta method with time-step $h=10^{-8}$ is used to solve the two systems of differential equations (3) and (4) with the active nonlinear controller (7). We take $k_{i}=4$ for $i=1,2,3$.

The parameters of the Wang four-scroll systems are chosen so that the systems (3) and (4) are chaotic, ie

$$
a=1, \quad b=5.7, \quad c=5, \quad d=0.06 .
$$

The initial values of the master system (3) are chosen as

$$
x_{1}(0)=12, \quad x_{2}(0)=-9, \quad x_{3}(0)=8 .
$$

The initial values of the slave system (4) are chosen as

$$
y_{1}(0)=-2, \quad y_{2}(0)=15, \quad y_{3}(0)=20 \text {. }
$$

Figure 3 shows the hybrid synchronization of the Wang four-scroll systems (3) and (4). Figure 4 shows the timehistory of the hybrid synchronization errors.

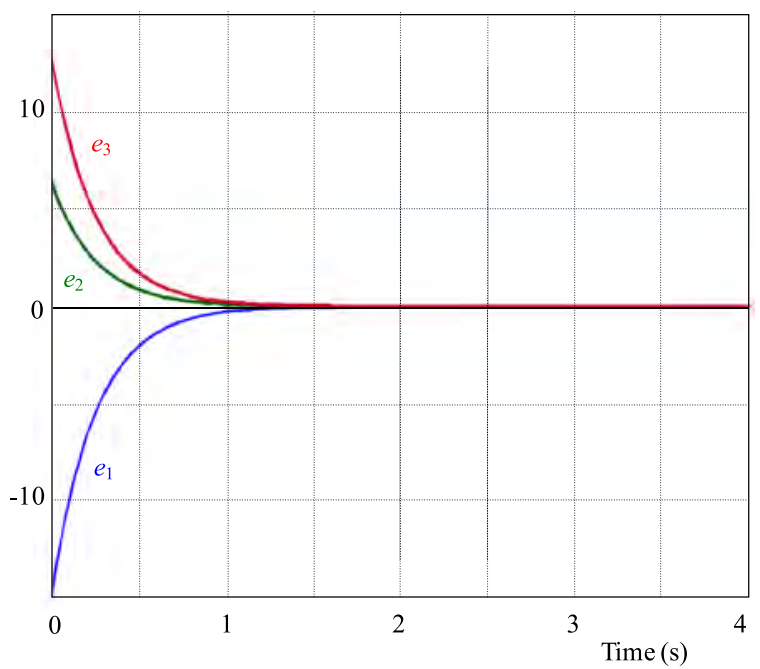

Fig. 4. History of the error states $e_{1}, e_{2}, e_{3}$ 

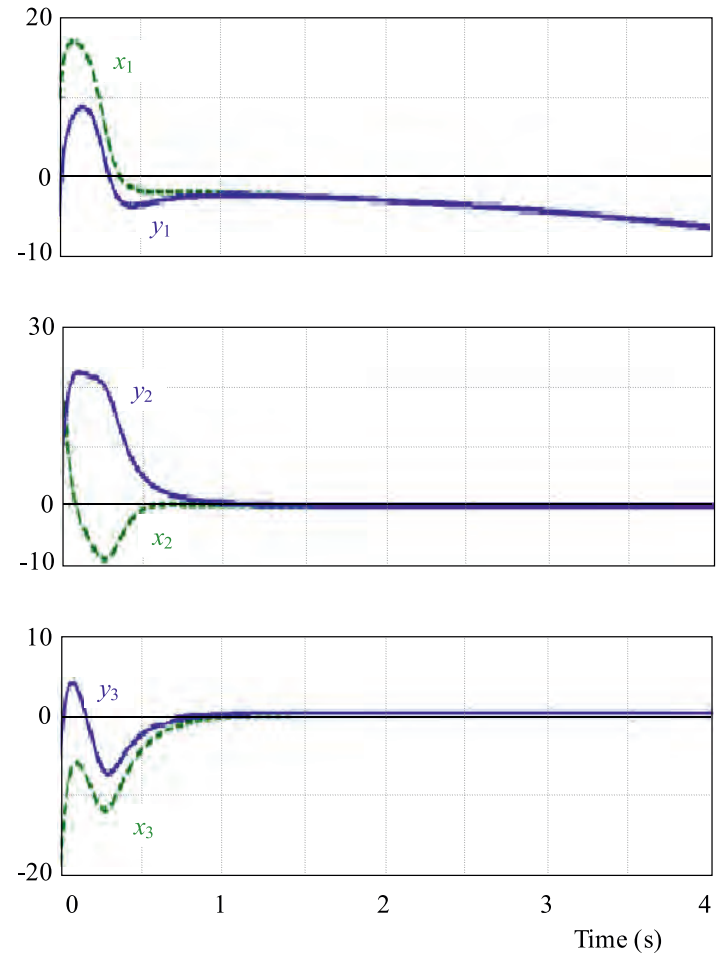

Fig. 5. Hybrid synchronization of the identical Liu-Chen four-scroll systems

\section{HYBRID SYNCHRONIZATION OF THE IDENTICAL LIU-CHEN FOUR-SCROLL SYSTEMS}

\subsection{Theoretical Results}

In this section, we deploy the active control method to derive new results for the hybrid synchronization of identical Liu-Chen four-scroll systems ([43], 2004).

Thus, the master system is described by the Liu-Chen dynamics

$$
\begin{aligned}
& \dot{x}_{1}=\alpha x_{1}-x_{2} x_{3}, \\
& \dot{x}_{2}=-\beta x_{2}+x_{1} x_{3}, \\
& \dot{x}_{3}=-\gamma x_{3}+x_{1} x_{2}
\end{aligned}
$$

where $x_{1}, x_{2}, x_{3}$ are the states and $\alpha, \beta, \gamma$ are positive, constant parameters of the system.

The slave system is described by the controlled Wang dynamics

$$
\begin{aligned}
& \dot{y}_{1}=\alpha y_{1}-y_{2} y_{3}+u_{1}, \\
& \dot{y}_{2}=-\beta y_{2}+y_{1} y_{3}+u_{2}, \\
& \dot{y}_{3}=-\gamma y_{3}+y_{1} y_{2}+u_{3}
\end{aligned}
$$

where $y_{1}, y_{2}, y_{3}$ are the states and $u_{1}, u_{2}, u_{3}$ are the active controllers to be designed.
The hybrid synchronization error is defined as

$$
\begin{aligned}
& e_{1}=y_{1}-x_{1}, \\
& e_{2}=y_{2}+x_{2}, \\
& e_{3}=y_{3}-x_{3} .
\end{aligned}
$$

A simple calculation gives the error dynamics as

$$
\begin{aligned}
& \dot{e}_{1}=\alpha e_{1}-y_{2} y_{3}+x_{2} x_{3}+u_{1}, \\
& \dot{e}_{2}=-\beta e_{2}+y_{1} y_{3}+x_{1} x_{3}+u_{2}, \\
& \dot{e}_{3}=-\gamma e_{3}+y_{1} y_{2}-x_{1} x_{2}+u_{3} .
\end{aligned}
$$

We define the active controls and as

$$
\begin{aligned}
& u_{1}(t)=-\alpha e_{1}+y_{2} y_{3}-x_{2} x_{3}-k_{1} e_{1}, \\
& u_{2}(t)=\beta e_{2}-y_{1} y_{3}-x_{1} x_{3}-k_{2} e_{2}, \\
& u_{3}(t)=\gamma e_{3}-y_{1} y_{2}+x_{1} x_{2}-k_{3} e_{3}
\end{aligned}
$$

where $k_{1}, k_{2}, k_{3}$ are positive constants.

Substituting (15) into (14), the error dynamics simplifies to

$$
\begin{aligned}
& \dot{e}_{1}=-k_{1} e_{1}, \\
& \dot{e}_{2}=-k_{2} e_{2}, \\
& \dot{e}_{3}=-k_{3} e_{3} .
\end{aligned}
$$

TheOREM 2. The identical Liu-Chen four-scroll systems (11) and (12) with constant, positive parameters are globally and exponentially hybrid-synchronized by the active control law (15), where the gains $k_{1}, k_{2}, k_{3}$ are positive constants.

Pro of. This result is a simple consequence of the Lyapunov stability theory.

Consider the quadratic Lyapunov function

$$
V\left(e_{1}, e_{2}, e_{3}\right)=\frac{1}{2}\left(e_{1}^{2}+e_{2}^{2}+e_{3}^{2}\right),
$$

which is a positive definite function on $R^{3}$.

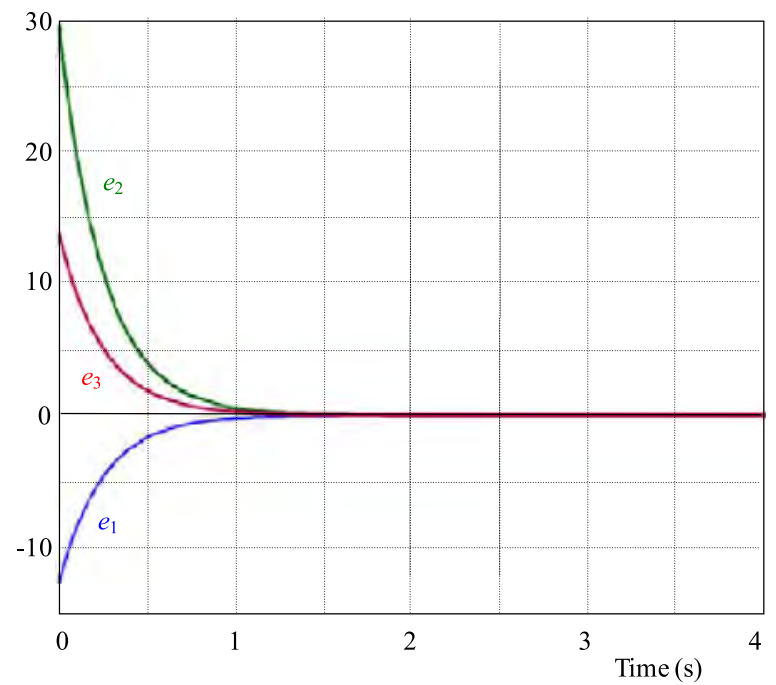

Fig. 6. History of the error states $e_{1}, e_{2}, e_{3}$ 
Differentiating $V$ along the trajectories of (16), we get

$$
\dot{V}=-k_{1} e_{1}^{2}-k_{2} e_{2}^{2}-k_{3} e_{3}^{2}
$$

which is a negative definite function on $R^{3}$. Hence, by the Lyapunov stability theory [44], it follows that $e_{i}(t) \rightarrow 0$ as $t \rightarrow \infty$ for $i=1,2,3$. This completes the proof.

\subsection{Numerical Results}

For the numerical simulations, the fourth-order RungeKutta method with time-step $h=10^{-8}$ is used to solve the two systems of differential equations (11) and (12) with the active nonlinear controller (15). We take $k_{i}=4$ for $i=1,2,3$. The parameters of the Liu-Chen fourscroll systems are chosen so that the systems (11) and (12) are chaotic, ie

$$
\alpha=0.4, \quad \beta=12, \quad \gamma=5 \text {. }
$$

The initial values of the master system (11) are chosen as

$$
x_{1}(0)=7, \quad x_{2}(0)=20, \quad x_{3}(0)=-19 .
$$

The initial values of the slave system (12) are chosen as

$$
y_{1}(0)=-6, \quad y_{2}(0)=10, \quad y_{3}(0)=-5 \text {. }
$$

Figure 5 shows the hybrid synchronization of the LiuChen four-scroll systems (11) and (12). Figure 6 shows the time-history of the hybrid synchronization errors.

\section{HYBRID SYNCHRONIZATION OF THE NON-IDENTICAL WANG AND LIU-CHEN FOUR-SCROLL SYSTEMS}

\subsection{Theoretical Results}

In this section, we deploy the active control method to derive new results for the hybrid synchronization of non-identical four-scroll systems, viz. Wang system ([42], 2009) and Liu-Chen system ([43], 2004).

Thus, the master system is described by the Wang dynamics

$$
\begin{aligned}
& \dot{x}_{1}=a\left(x_{1}-x_{2}\right)-x_{2} x_{3}, \\
& \dot{x}_{2}=-b x_{2}+x_{1} x_{3}, \\
& \dot{x}_{3}=-c x_{3}+d x_{1}+x_{1} x_{2}
\end{aligned}
$$

where $x_{1}, x_{2}, x_{3}$ are the states and $a, b, c$ are positive, constant parameters of the system.

The slave system is described by the controlled LiuChen dynamics

$$
\begin{aligned}
& \dot{y}_{1}=\alpha y_{1}-y_{2} y_{3}+u_{1}, \\
& \dot{y}_{2}=-\beta y_{2}+y_{1} y_{3}+u_{2}, \\
& \dot{y}_{3}=-\gamma y_{3}+y_{1} y_{2}+u_{3}
\end{aligned}
$$

where $y_{1}, y_{2}, y_{3}$ are the states, $\alpha, \beta, \gamma$ are positive, constant parameters of the system and $u_{1}, u_{2}, u_{3}$ are the active controllers to be designed.

The hybrid synchronization error is defined as

$$
\begin{aligned}
& e_{1}=y_{1}-x_{1}, \\
& e_{2}=y_{2}+x_{2}, \\
& e_{3}=y_{3}-x_{3} .
\end{aligned}
$$

A simple calculation gives the error dynamics as

$$
\begin{aligned}
& \dot{e}_{1}=\alpha y_{1}-a\left(x_{1}-x_{2}\right)-y_{2} y_{3}+x_{2} x_{3}+u_{1}, \\
& \dot{e}_{2}=-\beta y_{2}-b x_{2}+y_{1} y_{3}+x_{1} x_{3}+u_{2}, \\
& \dot{e}_{3}=-\gamma y_{3}+c x_{3}-d x_{1}+y_{1} y_{2}-x_{1} x_{2}+u_{3} .
\end{aligned}
$$

We define the active controls $u_{1}(t), u_{2}(t)$ and $u_{3}(t)$ as

$$
\begin{aligned}
& u_{1}(t)=-\alpha y_{1}+a\left(x_{1}-x_{2}\right)+y_{2} y_{3}-x_{2} x_{3}-k_{1} e_{1}, \\
& u_{2}(t)=\beta y_{2}+b x_{2}-y_{1} y_{3}-x_{1} x_{3}-k_{2} e_{2}, \\
& u_{3}(t)=\gamma y_{3}-c x_{3}+d x_{1}-y_{1} y_{2}+x_{1} x_{2}-k_{3} e_{3}
\end{aligned}
$$

where $k_{1}, k_{2}, k_{3}$ are positive constants.

Substituting (23) into (22), the error dynamics simplifies to

$$
\begin{aligned}
& \dot{e}_{1}=-k_{1} e_{1}, \\
& \dot{e}_{2}=-k_{2} e_{2}, \\
& \dot{e}_{3}=-k_{3} e_{3} .
\end{aligned}
$$

TheOREM 3. The non-identical Wang four-scroll system (19) and Liu-Chen four-scroll system (20) with constant, positive parameters are globally and exponentially hybrid-synchronized by the active control law (23), where the gains $k_{1}, k_{2}, k_{3}$ are positive constants.

Pro of. This result is a simple consequence of the Lyapunov stability theory. Consider the quadratic Lyapunov function defined by

$$
V\left(e_{1}, e_{2}, e_{3}\right)=\frac{1}{2}\left(e_{1}^{2}+e_{2}^{2}+e_{3}^{2}\right)
$$

which is a positive definite function on $R^{3}$.

Differentiating $V$ along the trajectories of (24), we get

$$
\dot{V}=-k_{1} e_{1}^{2}-k_{2} e_{2}^{2}-k_{3} e_{3}^{2}
$$

which is a negative definite function on $R^{3}$. Hence, by the Lyapunov stability theory [44], it follows that $e_{i}(t) \rightarrow 0$ as $t \rightarrow \infty$ for $i=1,2,3$. This completes the proof. 

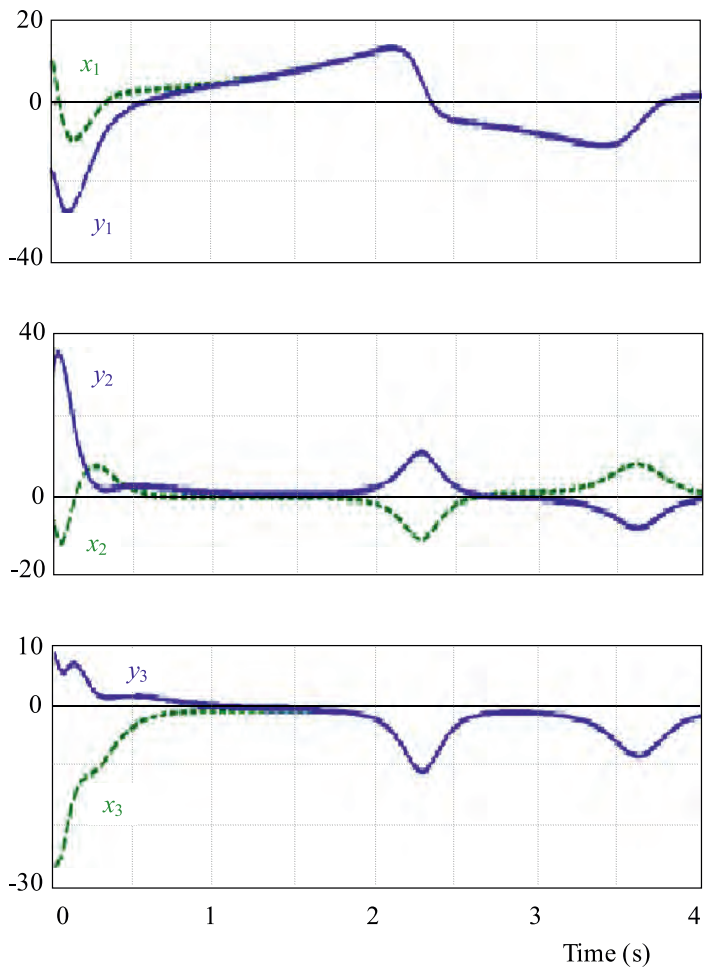

Fig. 7. Hybrid synchronization of the non-identical Wang and LiuChen four-scroll systems

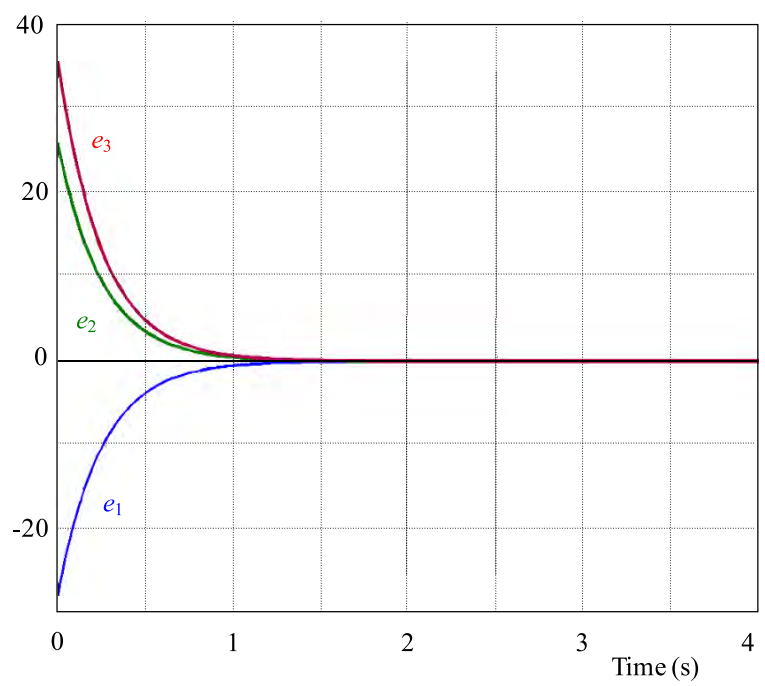

Fig. 8. History of the error states $e_{1}, e_{2}, e_{3}$

\subsection{Numerical Results}

For the numerical simulations, the fourth-order RungeKutta method with time-step $h=10^{-8}$ is used to solve the two systems of differential equations (19) and (20) with the active nonlinear controller (23).

We take $k_{i}=4$ for $i=1,2,3$.

The parameters of the Wang and Liu-Chen four-scroll systems are chosen as in the chaotic case, $i e$

$$
\begin{gathered}
a=1, \quad b=5.7, \quad c=5, \quad d=0.06, \\
\alpha=0.4, \quad \beta=12, \quad \gamma=5 .
\end{gathered}
$$

The initial values of the master system (19) are chosen as

$$
x_{1}(0)=12, \quad x_{2}(0)=-4, \quad x_{3}(0)=-27 .
$$

The initial values of the slave system (20) are chosen as

$$
y_{1}(0)=-16, \quad y_{2}(0)=30, \quad y_{3}(0)=9 .
$$

Figure 7 shows the hybrid synchronization of the Wang system (19) and the Liu-Chen system (20). Figure 8 shows the time-history of the hybrid synchronization errors.

\section{CONCLUSIONS}

In this study, we have applied active control method for the hybrid chaos synchronization of the identical Wang four-scroll systems (2009), the identical Liu-Chen four-scroll systems (2004) and non-identical Wang and Liu-Chen four-scroll systems. The hybrid synchronization results derived in this paper have been proved using the Lyapunov stability theory. Since the Lyapunov exponents are not required for these calculations, the proposed adaptive control method is very effective and convenient for achieving hybrid synchronization of the four-scroll systems addressed in this paper. Numerical simulations are shown to validate and illustrate the hybrid synchronization results derived in this paper for the four-scroll chaotic systems.

\section{REFERENCES}

[1] Alligood, K. T.-SAUER, T.-YORKE, J. A.: An Introduction to Dynamical Systems, Springer, New York, USA, 1997.

[2] LORENZ, E. N.: Deterministic Nonperiodic Flow, Journal of the Atmospheric Sciences 20 No. 2 (1963), 130-141.

[3] LAKSHMANAN, M.-MURALI, K.: Chaos in Nonlinear Oscillators: Controlling and Synchronization, World Scientific, Singapore, 1996..

[4] HAN, S. K.-KERRER, C.-KURAMOTO, Y.: D-Phasing and Bursting in Coupled Neural Oscillators, Physical Review Letters 75 (1995), 3190-3193.

[5] BLAsiUs, B.-HUPPERT, A.-STONE, L. : Complex Dynamics and Phase Synchronization in Spatially Extended Ecological System, Nature 399 (1999), 354-359.

[6] CUOMO, K. M.-OPPENHEIM, A. V.-STROGATZ, S. H.: Synchronization of Lorenz-Based Chaotic Cicuits with Applications to Communications, IEEE Trans. Circuits and Systems II 40 (1993), 626-633.

[7] KOCAREV, L.-PARTLIZ, U.: General Approach for Chaotic Synchronization with Applications to Communications, Physical Review Letters 74 (1995), 5028-5030.

[8] LU, J.-WU, X.-LÜ, J. : Synchronization of a Unified Chaotic System and the Application in Secure Communication, Physics Letters A 305 (2002), 365-370.

[9] PECORA, L. M.-CARROLL, T. L.: Synchronization in Chaotic Systems, Physical Review Letters 64 No. 8 (1990), 821-824.

[10] OTT, E.-GREBOGI, C. YORKE, J. A.: Controlling Chaos, Physical Review Letters 64 (1990), 1196-1199. 
[11] YANG, T.-CHUA, L. O.: Control of Chaos using Sampled-Data Feedback Control, International Journal of Bifurcation and Chaos 9 (1999), 215-219.

[12] PARK, J. H.-KWON, O. M. : A Novel Criterion for Delayed Feedback Control of Time-Delay Chaotic Systems, Chaos, Solitons and Fractals 17 (2003), 709-716.

[13] HUA, C.-GUAN, X. : Robust Control of Time-Delay Chaotic Systems, Physics Letters A $\mathbf{3 1 4}$ (2003), 72-80.

[14] HO, M. C.-HUNG, Y. C. : Synchronization of Two Different Chaotic Systems using Generalized Active Control, Physics Letters A 301 (2002), 424-428.

[15] YASSEN, M. T.: Chaos Synchronization between Two Different Chaotic Systems using Active Control, Chaos, Solitons and Fractals 23 (2005), 131-140.

[16] TIAN, L.-XU, J.-SUN, M.: Chaos Synchronization of the Energy Resource Chaotic System with Active Control, International Journal of Nonlinear Science 3 No. 3 (2007), 228-234.

[17] SUNDARAPANDIAN, V.: Global Chaos Synchronization of Shimizu-Morioka and Liu-Chen Chaotic Systems by Active Nonlinear Control, International Journal of Advances in Science and Technology 2 No. 4 (2011), 11-20.

[18] SUNDARAPANDIAN, V.: Global Chaos Synchronization of Liu and Harb Chaotic Systems by Active Nonlinear Control, International Journal of Computer Information Systems 2 No. 5 (2011), 8-12.

[19] YASSEN, M. T.: Applied Mathematics and Computation 135 (2001), 113-120.

[20] CHEN, S. H.-LÜ, J. : Synchronization of an Uncertain Unified System via Adaptive Control, Chaos, Solitons and Fractals 14 (2002), 643-647.

[21] JIA, L.-TONG, H.: Adaptive Control and Synchronization of a Four-Dimensional Energy Resources System of JiangSu Province, International Journal of Nonlinear Science 7 No. 3 (2009), 307-309.

[22] SUNDARAPANDIAN, V.: Adaptive Synchronization of Uncertain Sprott H and I Chaotic Systems, International Journal of Computer Information Systems 2 No. 5 (2011), 1-7.

[23] SUNDARAPANDIAN, V.: Adaptive Control and Synchronization of the Shaw Chaotic System, International Journal in Foundations of Computer Science and Technology 1 No. 1 (2011) $1-11$.

[24] MASCOLO, S.-GRASSI, G.: Controlling Chaotic Dynamics using Backstepping Design with Application to the Lorenz System and Chua's Circuit, International Journal of Bifurcation and Chaos 9 (1999), 1425-1434.

[25] TAN, X.-ZHANG-YANG, Y.: Synchronizing Chaotic Systems using Backstepping Design, Chaos, Solitons and Fractals 16 (2003), 37-45.

[26] ZHANG, H.-MA, X.-LI, M.-ZOU, J. : Controlling and Tracking Hyperchaotic Rossler System via Active Backstepping Design, Chaos, Solitons and Fractals 26 (2005), 353-361.

[27] UTKIN, V. I. : Variable Structure Systems using Sliding Mode, IEEE Transactions on Automatic Control 22 (1977), 212-222.

[28] SLOTINE, J. E.-SASTRY, S. S. : Tracking Control of Nonlinear Systems using Sliding Surface with Application to Robotic Manipulators, International Journal of Control 38 (1983), 465-492.

[29] SUNDARAPANDIAN, V.-SIVAPERUMAL, S.: Global Chaos Synchronization of the Hyperchaotic Qi Systems by Sliding Mode Control, International Journal on Computer Science and Engineering 3 No. 6 (2011), 2430-2437.

[30] SUNDARAPANDIAN, V.: Global Chaos Synchronization of the Pehlivan Systems by Sliding Mode Control, International Journal on Computer Science and Engineering 3 No. 5 (2011), $2163-2169$.
31] GE, Z. M.-CHEN, C. C.: Phase Synchronization of Coupled Chaotic Multiple Time Scales Systems, Chaos, Solitons and Fractals 20 (2004), 639-647.

32] WANG, Y. W.-GUAN, Z. H.: Generalized Synchronization of Continuous Chaotic Systems, Chaos, Solitons and Fractals 27 (2006), 97-101

[33] ZHANG, X.-ZHU, H. : Anti-Synchronization of Two Different Hyperchaotic Systems via Active and Adaptive Control, Interntaional Journal of Nonlinear Science 6 (2008), 216-223.

34] CHIANG, T.-LIN, J.-LIA, T.-YAN, J. : Anti-Synchronization of Uncertain Unified Chaotic Systems with Dead-Zone Nonlinearity, Nonlinear Analysis 68 (2008), 2629-2637.

35] SUNDARAPANDIAN, V.: Anti-Synchronization of Arneodo and Coullet Systems by Active Nonlinear Control, International Journal on Control Theory and Applications 4 No. 1 (2011), $25-36$.

36] SUNDARAPANDIAN, V.-SIVAPERUMAL, S. : Anti-Synchronization of Hyperchaotic Lorenz Systems by Sliding Mode Control, International Journal on Computer Science and Engineering 3 No. 6 (2011), 2450-2457.

37] QIANG, J.: Projective Synchronization of a New Hyperchaotic Lorenz System, Physics Letters A 370 (2007), 40-45.

[38] JIAN-PING, Y.-CHANG-PIN, L.: Generalized Projective Synchronization for the Chaotic Lorenz System and the Chaotic Chen System, J. Shanghai University 10 (2006), 299-304.

[39] LI, R. H.-XU, W.-LI, S. : Adaptive Generalized Projective Synchronization in Different Chaotic Systems based on Parameter Identification, Physics Letters A 367 (2007), 199-206.

[40] SARASU, P.-SUNDARAPANDIAN, V.: Active Controller Design for Generalized Projective Synchronization of Four-Scroll Chaotic Systems, International Journal of System Signal Control and Engineering Application 4 No. 2 (2011), 26-33.

[41] SUNDARAPANDIAN, V.: Hybrid Synchronization of Lorenz and Pehlivan Chaotic Systems by Active Nonlinear Control, International Journal of Advances in Science and Technology 2 No. 6 (2011), 10-20.

42] WANG, L.: 3-Scroll and 4-Scroll Chaotic Attractors Generated from a New 3-D Quadratic Autonomous System, Nonlinear Dynamics 56 (2009), 453-462.

[43] LIU, W.-CHEN, G.: Can a Three-Dimensional Smooth Autonomous Quadratic Chaotic System Generate a Single Four-Scroll Attractor?, International J. Bifur. Chaos 14 (2004), 1395-1403.

[44] HAHN, W.: The Stability of Motion, Springer, New York, USA, 1967.

Received 25 February 2013,

Karthikeyan Rajagopal is a Professor and the Head of the Department of Electronics and Communication Engineering at Velammal Institute of Technology, Chennai, India since 2012. He has 14 publications in Scopus-indexed journals and conferences. His areas of interest include Wireless Networks, Security Algorithms, Embedded Systems and Control Systems.

Sundarapandian Vaidyanathan is a Professor and the Dean at the Research and Development Centre, Vel Tech Dr RR \& Dr SR Technical University, Chennai, India. His current research focuses on Control Systems, Chaos Theory and Computational Science. He has published over 100 Scopus-indexed research publications. He has delivered many plenary talks on Control Engineering and Chaos in international conferences. 